\title{
El ecoturismo como estrategia de desarrollo rural en América Latina. Caso de la aldea indígena Plan Grande Quehueche (Izabal, Guatemala)
}

\author{
Francisco Enríquez Narváez* y Rafael Blanco Sepúlveda**
}

\begin{abstract}
RESUMEN
ABSTRACT

Los proyectos de desarrollo rural a escala internacional ponen cada vez mayor énfasis en la implementación de proyectos de ecoturismo, aprovechando como recursos los valores culturales y ambientales del territorio. Los resultados, a nivel general, no se pueden considerar satisfactorios. En este trabajo se analizan las causas de los fracasos de muchos de los proyectos de ecoturismo que se han implementado en América Latina, para profundizar después en los casos que se han desarrollado en Guatemala, abriendo

una puerta ai optimismo a partir de los resultados que se están obteniendo en un proyecto de ecoturismo que se desarrolla en la aldea Plan Grande Quehueche de Izabal (Guatemala).

Rural development projects, at international level, are based everincreasing in the implementation of ecotourism projects. This tourism type is supported by the rich cultural and ecological values existing in the territory. The results, in general, cannot be considered satisfactory. In this paper the failures causes of many ecotourism projects implemented in Latin America, are analyze, afterwards the cases that have developed in Guatemala and finally the good results obtained in the ecotourism project of the small village Plan Grande Quehueche of the Department of Izabal in Guatemala are also analyzed.

PALABRAS CLAVE: ecoturismo, desarrollo rural, América

KEY WORDS: ecotourism, rural development, Latin Latina. America.
\end{abstract}

* Organización Internacional del Trabajo (OIT) en Guatemala. Miembro de la Asociación Ak' Tenamit, Izabal (Guatemala).

** Departamento de Geografía. Universidad de Málaga. 


\section{INTRODUCCIÓN}

El Desarrollo Rural, en la actualidad, ha dejado de ser sinónimo de desarrollo agrícola. Esta actividad es ahora un componente más de la economía rural, siendo el uso múltiple del territorio la tendencia actual en desarrollo rural. El nuevo marco de trabajo se basa en el fomento de nuevas actividades económicas, aprovechando para ello los recursos endógenos, tanto medioambientales como culturales.

La multifuncionalidad del espacio rural es el modelo de desarrollo que se está siguiendo en la actualidad en las comunidades rurales de los países en vías de desarrollo. En este sentido, hay que destacar que el turismo es una de las actividades económicas más importantes a nivel internacional y, asimismo, es la línea de trabajo en Desarrollo Rural que más ha crecido en los últimos años, no sólo en América Latina, sino también en el resto del mundo. A esta escala, la actividad turística representa el $12 \%$ del PNB mundial y emplea a 1 de cada 12 trabajadores del planeta (Newsome et al., 2002).

El turismo convencional ha sido incapaz, hasta el momento, de impulsar el desarrollo de las comunidades rurales, en primer lugar, porque se desarrolla sin involucrar a la población local y en segundo lugar, porque es una actividad generadora de impactos negativos, tanto sobre el medio natural como sobre las culturas locales (Mason, 2003). Por este motivo, se han impulsado en los planes de desarrollo rural un turismo alternativo, enfocado desde la perspectiva de la sostenibilidad (Weaber, 1988; Farell y Runyan, 1991; Honey, 1999; Wearing y Neil, 1999). Bajo el concepto de turismo sostenible se engloban las siguientes modalidades de turismo: agroturismo, etnoturismo, turismo verde, rural, científico y de aventuras, entre otras modalidades (Novelli, 2004). Todas ellas se pueden considerar acepciones que comúnmente se engloban bajo el término ecoturismo. Esta modalidad incorpora una nueva concepción del turismo como industria y del turista como cliente, porque promociona la sensibilidad hacia la naturaleza, la cultura y las formas de vida de las poblaciones receptoras. Las dos cuestiones esenciales del ecoturismo son: la participación de la población local y el respeto al medio ambiente y los valores culturales, como únicas vías para asegurar a largo plazo un desarrollo turístico sostenible (Grenier et al., 1993; Ross y Wall, 1999; Masberg y Morales, 1999; Burger, 2000; Nello, 2003), teniendo en cuenta, además, que la generación de beneficios económicos no se puede valorar siguiendo el sistema financiero convencional porque, en este caso, el ecoturismo sería considerado como una modalidad de turismo de escasa rentabilidad económica (Lindberg y Enriquez, 1996).

Se han sucedido a lo largo de las últimas décadas varias cumbres internacionales sobre turismo que han tratado como tema central de sus actos la sostenibilidad de esta actividad. En la Conferencia de Estocolmo (1972) y en las Cumbres de Río de Janeiro (1992) y Johannesburgo (2002) se propugna un cambio en el modelo actual de desarrollo para avanzar en el camino de la sostenibilidad y alcanzar un desarrollo social, económico y ambiental equilibrado del territorio. Del mismo modo, el desarrollo sostenible aplicado al turismo es la línea de trabajo que 
se marcó en la Conferencia de la Haya, celebrada en 1989, de la que se extrae una declaración con clara vocación de instrumento de cooperación internacional. Los encuentros en esta materia se han ido sucediendo, como la Declaración de Berlín de 1997 en la que se establecieron las formas sostenibles de turismo y se dejaron sentadas las bases para la aprobación posterior de un acta sobre turismo y diversidad biológica, vinculada al derecho internacional. También se pueden destacar los encuentros que han tenido un carácter más regional, como la Conferencia Internacional sobre Turismo y Desarrollo Sostenible en el Mediterráneo, celebrada en 1997 en Calvià (Mallorca). Con respecto al ecoturismo, se han venido celebrando conferencias internacionales desde mediados de la década de los 90 . Más recientemente, y con motivo de la celebración del año internacional de Ecoturismo (2002), el Programa de las Naciones Unidas para el Medio Ambiente (PNUMA) y la Organización Mundial del Turismo (OMT) celebraron en Québec (Canadá) la Cumbre Mundial del Ecoturismo, con el objetivo de preparar el programa preliminar para la Cumbre Mundial sobre el Desarrollo Sostenible (CMDS) de Johannesburgo, antes citada.

\section{EL ECOTURISMO EN AMÉRICA LATINA}

Las bases fundamentales sobre las que se sustenta el ecoturismo son los ricos valores ecológicos y culturales que existen en el territorio. En América Latina existe una elevada biodiversidad natural, junto con una rica y variada cultura indigena, que se encuentra en la actualidad, en condiciones muy dispares. Asi por ejemplo, en relación a los valores culturales, en Guatemala se conservan, a grandes rasgos, gran parte de los valores tradiciones ya que el proceso de aculturación, desde la colonización española, ha sido bastante limitado; mientras que, en Nicaragua, Costa Rica o Argentina la pervivencia de la cultura tradicional es muy reducida.

La mayor parte de los primeros proyectos de ecoturismo que se implementaron en América Latina se han quedado en un conjunto de buenas intenciones que no han llegado a materializarse (Luck y Kirstages, 2002). En primer lugar, porque estos proyectos estaban mal planificados, es decir se realizaron sin estudios de capacidad de carga, lo que provocó graves impactos sobre la biodiversidad y las poblaciones indígenas. Las causas de estos problemas son, en primer lugar, el contexto de vacío jurídico en materia turística y medioambiental en estos países (Nello, 2003). Y, en segundo lugar, porque estos proyectos no se suelen implementar para beneficiar directamente a la población local.

Costa Rica es un buen ejemplo de este fenómeno. Steam (2001), Morera (2002), Nello (2003) y Solano (2003) han puesto de manifiesto que este país cuenta con numerosas áreas naturales protegidas y con excelentes profesionales en medio ambiente y turismo, pero los resultados no han sido del todo satisfactorios para la población rural porque los principales beneficiarios de esta actividad 
han sido los propietarios de los complejos turísticos que, en la mayoría de los casos, son de capital extranjero. Pese a todo ello, hay que reconocer que este pais, junto con Belice, cuenta con una de las economías más saneadas de toda Centroamérica, lo cual se debe en gran parte al ecoturismo. Según datos de la IX Cumbre Iberoamericana de Jefes de Estado y Gobierno, celebrado en La Habana en 1999, el ecoturismo ${ }^{1}$ constituye la primera fuente de ingresos de Costa Rica, por delante de la industria textil y la producción de café y banano, los productos tradicionales del país, representando el 10,14\% de su PIB en el año 1997, y con un crecimiento del 17\% anual entre los años 1990 y 1995.

Otro caso ilustrativo, en el que los proyectos ecoturísticos no han tenido el éxito esperado ha sido puesto de manifiesto por Wallace y Pierce (1996) para Brasil. Estos autores indican que el ecoturismo en este país ha contribuido poco a la protección de los recursos y no se ha preocupado por la habilitación y participación de la población local en estos proyectos.

Paralelamente a este proceso de desarrollo turístico, también existen en América Latina magníficos ejemplos de proyectos ecoturísticos que están dirigidos hacia la población local, lo que incluye también a la indígena. Desde la perspectiva del desarrollo, el ecoturismo sólo se puede considerar satisfactorio si la población local está implicada, ejerciendo algún tipo de control sobre dichos proyectos y beneficiándose directamente de los ingresos generados por esta actividad (Scheyvens, 1999). Los mejores casos se encuentran en Ecuador, cuna del turismo comunitario, donde existen algunos de los proyectos de este tipo más exitosos de todo el continente (Jacomé y Acosta, 2003; Bustos, 2003). Estos proyectos se han basado en el respeto a la cultura tradicional y a los recursos naturales, generando, al mismo tiempo, importantes ingresos para las comunidades kichuas. Los reconocimientos internacionales a estos proyectos son continuos y su organización se puede considerar, un modelo a imitar incluso en países desarrollados. Existen otros muchos casos, también exitosos, entre los que se pueden destacar los de Dominica (Weaber, 1991) y México (Foucat, 2002).

En todos estos casos, el turismo ha sido una via eficaz de desarrollo ya que ha proporcionado ingresos directamente a las comunidades aldeanas, siendo mínimas las pérdidas a través de los intermediarios. Este enfoque es diferente a los proyectos agropecuarios, de artesanía, etc., ya que en estos casos se generan productos que sufren un encarecimiento a través del proceso de comercialización. Por esta causa, el ecoturismo se ha convertido en una de las principales líneas de trabajo de la cooperación internacional en los países en vías de desarrollo. Sin embargo, se entiende que es fundamental la complementariedad económica de todos estos proyectos porque la multifuncionalidad del espacio rural es la mejor vía para alcanzar un desarrollo económico sostenible.

1 Las cifras estadísticas que se ofrecen en la Cumbre no diferencian el turismo convencional del ecoturismo. Para el caso de Costa Rica hay que indicar que este sector económico se encuadra principalmente en la modalidad de ecoturismo. 
El ecoturismo no suele trabajar con los operadores turísticos tradicionales debido a que las ofertas son muy diferentes, ya que se ofrece un destino turístico a grupos reducidos para minimizar el posible impacto ambiental y cultural. Por estos motivos, las agencias que trabajan con este tipo de ofertas son distintas y suelen además tener un enfoque más solidario, proporcionando a las comunidades beneficiarias del servicio turístico la mayor parte de los beneficios.

De todas formas, hay que indicar que hay determinados destinos internacionales, concretamente en algunos países asiáticas y de nuevo en Costa Rica, que son controlados por las agencias tradicionales de turismo, debido a que es un sector en alza y con grandes expectativas de mercado. En estos casos, no suelen existir limitaciones de capacidad de acogida por lo que estos destinos se ven desbordados por la masiva afluencia de turistas. Este hecho es contrario a los planteamientos del ecoturismo, propiamente dicho, sin embargo los destinos anteriores se siguen mal denominando ecoturísticos. Orams (1995) pone de manifiesto este hecho y señala que es necesario implementar mejores estrategias de manejo para alcanzar un modelo óptimo de turismo sostenible basado en el ecoturismo.

Al margen de las agencias de viajes, un gran volumen de turistas accede al ecoturismo a través de internet, haciendo sus reservas directamente con la empresa u organizaciones ecoturísticas, o llegan directamente al lugar del servicio en busca de alojamiento o las actividades ofertadas. Esta vía presenta la ventaja de que el mayor porcentaje de beneficios se queda con quien presta el servicio.

\section{EL ECOTURISMO EN EL CARIBE GUATEMALTECO (DEPARTAMENTO DE IZABAL, GUATEMALA)}

Una de las regiones de Guatemala con mayor potencial ecoturístico es la orilla caribeña del pais, concretamente el Departamento de Izabal (Figura 1). El área cuenta con varios espacios protegidos de inmenso valor en cuanto a biodiversidad, con caobas, orquídeas, manatíes, jaguares, tucanes, loros y un largo etcétera de especies animales y vegetales. Estos valores naturales se enriquecen con el alto valor cultural del área, donde conviven 2 culturas, la afro-caribeña denominada garifuna y la maya del grupo étnico q'eqchi'.

Existen actualmente en el área numerosas organizaciones trabajando en desarrollo rural, gracias al apoyo internacional. Estas organizaciones se pueden diferenciar entre gubernamentales y no gubernamentales. Entre las primeras, destacan las dos municipalidades del Departamento de Izabal (Livingston y Puerto Barrios), la Gobernación Departamental de Izabal, la Delegación Departamental del Ministerio de Ambiente y Recursos Naturales de Guatemala, la Delegación Departamental del Ministerio de Educación y el Consejo Nacional de Áreas Protegidas de Guatemala. $Y$ entre las no gubernamentales, se encuentran, entre otras: Defensores de la $\mathrm{Na}$ turaleza, Asociación Ak' Tenamit, Defensoría Maya-Q'eqchi', Fundación para el 


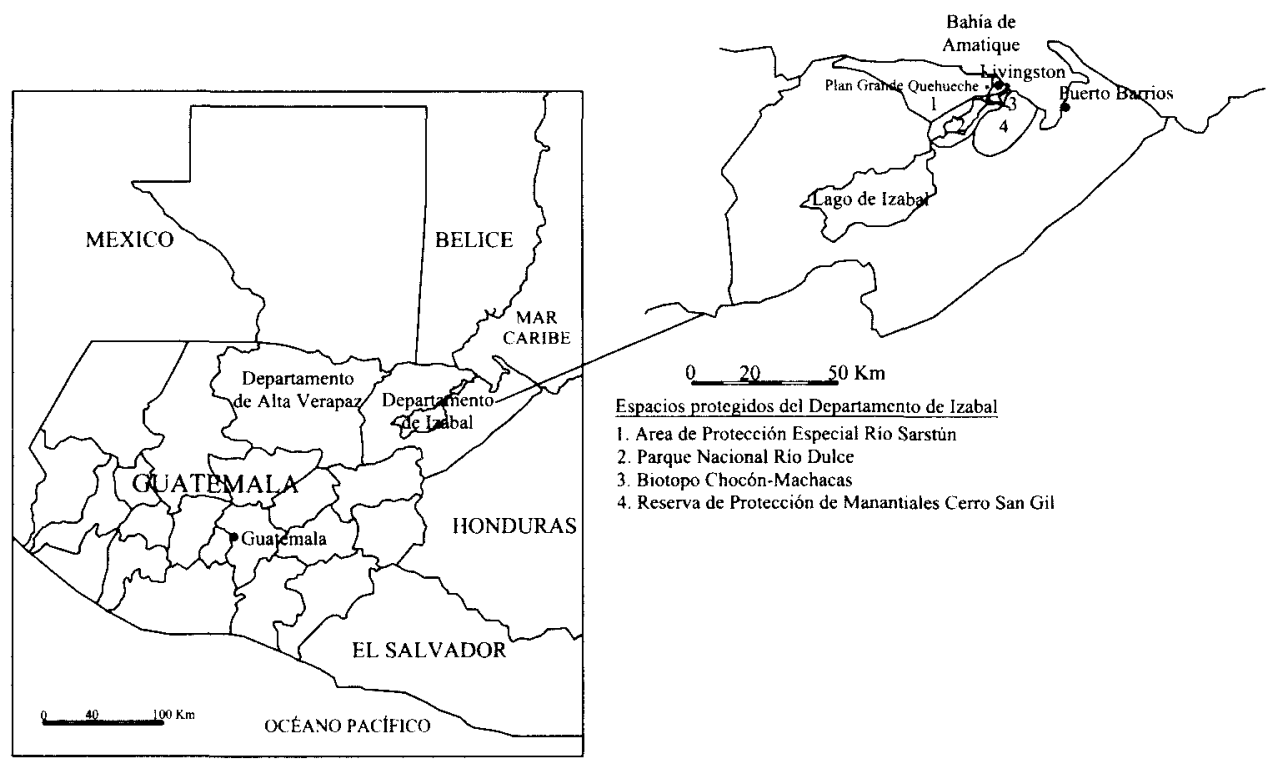

Figura 1. Mapa de localización de la zona de estudio.

Eco-desarrollo (FUNDAECO), Fundación Mario Dary, Delegación de Derechos Humanos, Proyecto RECOSMO y algunos comités de turismo locales.

Los resultados obtenidos hasta ahora se pueden considerar exiguos. Después de más de 15 millones de dólares invertidos en los últimos 10 años, el efecto sobre la situación económica de la población rural ha sido insignificante, incrementándose aun más la situación de pobreza en determinadas zonas. Las causas de los fracasos de estos proyectos son diversas. En unos casos, los proyectos de cooperación han tratado de aplicar modelos de desarrollo que han tenido éxito en los paises de origen de las organizaciones cooperantes, en zonas donde las condiciones, tanto ambientales como socio-económicas, son muy diferentes. Ésta ha sido una de las causas por la que muchos proyectos desarrollados en América Latina no han obtenido resultados positivos.

Greif $(1993,1994)$ indica que el crecimiento económico es un proceso complejo, en el que la organización de la sociedad juega un papel muy importante y esta sociedad es el reflejo de la compleja combinación de procesos económicos, políticos, culturales e históricos que han tenido lugar en el seno de dicha sociedad y que los individualizan de otras. De ahí se deriva la necesidad de contemplar cada caso de forma particular y no de aplicar programas de desarrollo de forma estándar.

En otros casos, la Cooperación Internacional ha fracasado cuando los proyectos de desarrollo rural se han basado en la construcción de infraestructuras y en las ayudas a la capacitación técnica y de gestión de los ayuntamientos y órganos de gobierno. La causa es que se ha partido de una base inadecuada, ya que se 
han obviado las situaciones legislativas y jurídicas de los paises en cuestión y la buena ejecución de los proyectos ha chocado con la corrupción de los cargos públicos. Esta ha sido una de las líneas de actuación de los organismos de Cooperación Internacional de varios países europeos, entre ellos España.

Hay ejemplos muy ilustrativos en Guatemala en los que la Cooperación Internacional apoyó a municipalidades, el equivalente a ayuntamientos, con proyectos dirigidos al fortalecimiento institucional, formando para ello técnicos cualificados y proporcionando mobiliario y equipos informáticos y electrónicos. Se construyeron además determinadas infraestructuras, depuradoras de agua y nuevos edificios que fueron destinados por ejemplo a programas sociales. En uno de estos casos, el proyecto de Cooperación Internacional terminó en un auténtico fracaso ${ }^{2}$. A raíz de unas elecciones municipales en las que se produjo un cambio de alcalde, se sucedieron determinadas circunstancias para que el proyecto fracasara:

a) Los equipos donados a la municipalidad desaparecieron.

b) Como consecuencia del cambio de autoridades en la municipalidad, el personal cualificado, al no pertenecer al cuerpo de funcionarios, fue despedido y otro personal no cualificado fue contratado.

c) Parte de los nuevos edificios que se construyeron fueron alquilados a un banco, dejando sin suficientes infraestructuras a los programas sociales que albergaba.

d) La depuradora está en desuso porque la municipalidad no tiene fondos para su mantenimiento.

La impunidad con que quedan estas acciones es flagrante, pero está justificada porque su denuncia puede ser objeto de represalias, lo que puede llegar a significar la muerte del denunciante. Para que esto no vuelva a ocurrir, estos tipos de proyectos deben partir de un conocimiento previo de la situación económica, social y jurídica del municipio. De esta forma, se podrían establecer las siguientes premisas de actuación:

a) La formación en personal es adecuada pero se debe partir asegurando la estabilidad laboral de dicho personal.

b) Las donaciones de material sin fortalecer previamente las instituciones jurídicas pueden tener los efectos señalados, por lo tanto, se debe partir del fortalecimiento de dichas instituciones.

c) Las infraestructuras necesitan un mantenimiento, por lo que se debió prever de donde iban a partir esos fondos. Si este estudio se hubiera realizado se habria llegado a la conclusión de que era necesario fortalecer el sistema de recaudación de impuestos de la municipalidad, dado el elevado fraude que existe.

${ }^{2}$ Los autores de este trabajo han decidido reservarse datos más específicos sobre la municipalidad de acogida del proyecto por razones de seguridad. 
Las experiencias que se tienen hasta el momento indican, que los proyectos más exitosos son los que los organismos de Cooperación Internacional han apoyado financieramente a las organizaciones de Desarrollo Rural del propio país, limitando su actuación a una labor de mera supervisión, para evitar el fraude o la malversación de fondos. La causa es que las organizaciones de Desarrollo Rural de los países en cuestión, tienen un mejor conocimiento de la realidad política, social y económica del país. El contacto diario con la realidad es el primer eslabón de una cadena que debe conducir al éxito de los proyectos de desarrollo. Los agentes de cooperación no pueden conocer los problemas del país a través de los medios de comunicación, es necesario que tengan un contacto real con los problemas, para desarrollar, de esta forma, proyectos adecuados a las necesidades reales de la población.

Las perspectivas de futuro son inciertas para la región, y en general para el país, porque después de casi 8 años de la firma de los acuerdos de paz, la cooperación internacional está comenzando a marcharse a otros países más necesitados y, los proyectos, al no ser la mayoría de ellos sostenibles, van a fracasar en el momento en el que todas estas ONGs dejen de tener fondos para apoyarlos.

\section{EL TRABAJO EN DESARROLLO RURAL DE LA ASOCIACIÓN AK' TENAMIT EN EL DEPARTAMENTO DE IZABAL (GUATEMALA)}

La Asociación Ak' Tenamit es una de las pocas organizaciones de Guatemala que basan su trabajo en fomentar el desarrollo sostenible de las comunidades rurales. La mayoría de las instituciones gubernamentales y no gubernamentales de Guatemala trabajan en desarrollo, pero no desde la perspectiva de la sostenibilidad, sus objetivos suelen ser conservacionistas o científicos por lo que no implican a la población en su manejo de forma sostenible. Sin embargo, en los últimos años, debido a la presión de los donantes internacionales, estas organizaciones se han visto obligadas a incluir a la población en sus planes de trabajo, con escaso éxito.

Ak' Tenamit trabaja con 30 aldeas de la comunidad maya-q'echi' del Departamento de Izabal (Guatemala) lo que, traducido en habitantes, supone aproximadamente unas 8.500 personas. El área en el que trabaja la organización se caracteriza por su aislamiento. A muchas aldeas sólo se puede acceder por vía acuática, tras la cual a veces hay que caminar hasta 5 horas por la selva para llegar a las comunidades. Las infraestructuras básicas, como electricidad o agua potable, casi son anecdóticas y las escuelas son muy precarias, ya que no superan la educación primaria y los docentes suelen estar mal capacitados.

La educación es el principal objetivo de la Asociación Ak' Tenamit. El propósito es formar a la población local para que ésta lidere el proceso de desarrollo económico sostenible de la región. En los acuerdos de paz de Guatemala, firmados en 1996, se pactó que toda la población rural tendría acceso a una educación adap- 
tada a sus verdaderas necesidades y en su lengua madre y no en la oficial, que es el español. Después de 8 años, el gobierno no ha comenzado todavía con la reforma educativa y Guatemala continua siendo el segundo país de América Latina con más población analfabeta, después de Haití.

Ak' Tenamit es la única organización en todo el pais que ha conseguido implementar un sistema educativo enfocado a las verdaderas necesidades rurales del área y además bilingüe, con la cosmovisión maya, la equidad de género y la protección del medio ambiente, como ejes principales. La asociación cuenta actualmente con un instituto básico en Bienestar Rural (estudiantes de $7 .^{\circ}$ a $9 .^{\circ}$ grado educativo), un perito en Bienestar Rural (10..$^{\circ}$ a $12 .^{\circ}$ grado educativo) y otro en Turismo Sostenible y además, existe un internado para dar la oportunidad a los jóvenes de la región de continuar con su educación secundaria hasta el nivel pre-universitario. Para el futuro se pretende llegar a un acuerdo con la Universidad del Valle de Guatemala, para contar con estudios superiores en las dos últimas carreras señaladas.

Además de este objetivo educacional, la asociación cuenta con proyectos de salud, artesanía, desarrollo comunitario, equidad de género, ecoturismo y conservación del medio ambiente.

La Asociación Ak' Tenamit basa su trabajo en una filosofía y en unas pautas metodológicas determinadas. La filosofía de trabajo de la asociación es no regalar nada a las comunidades indigenas, ya que éstas, por muy pobres que sean, siempre puede pagar con su mano de obra. De esta manera, la población local siente que las donaciones les pertenecen y no son una limosna que, con el paso del tiempo, dejan de valorarse y crea sentimientos de paternalismo. Por ello, a cambio de las infraestructuras necesarias para cada proyecto, se exige mano de obra gratuita para su implementación y para otras obras sociales, que no han de ser necesariamente en sus comunidades. En cuanto a las pautas metodológicas de trabajo, destaca que, previamente al desarrollo de cada proyecto, la asociación realiza una consulta a la comunidad con la que va a trabajar, para conocer bien sus necesidades y no ejecutar proyectos que no son necesarios o cuyos beneficiarios no están de acuerdo. Además, la Asociación trata siempre de que en todo el proceso de ejecución del proyecto estén implicados los propios miembros de la comunidad. Para ello, se capacita a la población para que el seguimiento del proyecto por parte de la ONG no sea indefinido y que, tras un período de tiempo aceptable, la población pueda continuar, por si sola, con el proyecto.

Con respecto al capítulo de la financiación, hay que señalar que Ak' Tenamit es la única organización que trabaja en el Caribe guatemalteco que ha conseguido en 5 años generar recursos propios, por un valor que asciende al $35 \%$ de su presupuesto anual, lo que equivale a 140.000 euros de un presupuesto total que se eleva a 400.000 euros. Estos recursos cubren todos los gastos administrativos, gran parte del seguimiento de los proyectos y una parte de las nuevas iniciativas. Esta autofinanciación parcial se ha conseguido a partir de dos proyectos productivos 
propios, como es el caso de un servicio de ecoturismo en la sede de la asociación y un restaurante en la ciudad de Livingston. El $65 \%$ del presupuesto restante es aportado por entidades de ayuda al desarrollo, tanto nacionales como internacionales y se destina principalmente a la financiación de los nuevos proyectos.

De los proyectos que está desarrollando la Asociación Ak' Tenamit se ha seleccionado para su análisis, por el éxito de los resultados que se están obteniendo, el proyecto de ecoturismo que se desarrolla en la aldea indígena Plan Grande Quehueche (Izabal, Guatemala).

\section{EL PROYECTO DE ECOTURISMO EN LA ALDEA PLAN GRANDE QUEHUECHE (IZABAL, GUATEMALA)}

\subsection{Los objetivos y la necesidad de un proyecto basado en el ecoturismo}

El Departamento de Medio Ambiente de la Asociación Ak' Tenamit inició en 1999 la planificación de un proyecto piloto de ecoturismo, con el objetivo de fomentar la diversidad económica de las aldeas guatemaltecas del Departamento de Izabal y poder dar, de esta forma, una solución a los problemas económicos de la población rural, originados a raíz del fuerte aumento de la población de los últimos años, lo que está teniendo no sólo graves consecuencias de desnutrición en la población, sino también severos impactos medioambientales en el área de influencia de cada una de las aldeas.

Para entender los problemas actuales de las poblaciones indigenas de la selva caribeña de Guatemala, hay que partir de la premisa que este espacio no estaba habitado antes de la década de los años 50 del siglo xx. Durante el conflicto armado que sacudió Guatemala a partir de 1960, la población campesina maya de Alta Verapaz, uno de los departamentos más castigados por el ejército, huyó de la persecución, refugiándose en el vecino departamento de Izabal (Figura 1), pero antes, concretamente durante la década de los años 40 y 50 , el interés de los terratenientes, en complicidad con el gobierno, por las tierras de la población indígena provocó una intensa presión sobre este colectivo que favoreció una primera migración hacia la zona caribeña que se encontraba despoblada y no tenía interés económico para los terratenientes.

Por aquella fecha, la falta de los servicios mínimos como salud y agua potable, provocaron que la mortalidad fuese muy alta, lo que mantenía una adecuada relación entre población y recursos. Mientras se mantuvo esta situación, el medio natural, aunque se vio afectado sensiblemente por el asentamiento humano, no sufrió graves impactos ambientales. La población de Guatemala ha experimentado, en los últimos años, un notable crecimiento. Según el Instituto Nacional de Estadística del país, entre 1994 y 2002 la población ha aumentado a una tasa de crecimiento anual superior al $2 \%$, lo que ha provocado que la población pase de $8,3 \mathrm{mi}$ - 
llones en 1994 hasta 11,2 millones en 2002. Las causas son la reducción paulatina de la mortalidad $(6,92 \%)$, aunque la tasa de mortalidad infantil sigue siendo muy alta $(47,03 \%)$ y el mantenimiento de altas tasas de natalidad $(35,05 \%)$. Teniendo en cuenta que la tasa de fecundidad de Guatemala es de 4,66 nacimientos por mujer (la tasa más alta de Latinoamérica), se estima que la población del país aumentará hasta 16,2 millones en 2010. Esta situación demográfica está generando una mayor presión sobre los recursos naturales, tanto vegetales como animales. Muchas de estas especies se encuentran en peligro de extinción (Cuadro 1) por lo que se están produciendo graves impactos ambientales. No existen, en la actualidad, fuentes estadísticas fiables que puedan atestiguar el crecimiento de la población indigena de Guatemala que vive en la selva debido, por una parte a la falta de medios del Estado y, por otra, a la desconfianza de la población indígena, por lo que aún si existieran estas fuentes los datos serían poco fiables.

\section{Cuadro 1. Especies vegetales y animales con valor comercial que} se encuentran en peligro de extinción en Guatemala

\begin{tabular}{l|l}
\hline \multicolumn{1}{c|}{ Especies vegetales } & \multicolumn{1}{c}{ Especies animales } \\
\hline Amapola (Pseudobombax ellipticum) & Jaguar (Panthera onca) \\
Caoba (Swietenia macrophylla) & Ocelote (Herpallorus yaguaroundi) \\
Cedro (Cedrela odorata) & Manati (Trichechus manatus) \\
Chico-zapote (Manikara zapota) & Loro cabeza azul (Amazona farinosa) \\
Guano (Sabal mexicana) & Lechuza (Ciccaba nigrolineata) \\
Hormigo (Plastymiscium dimorphandrum) & Halcón murcielaguero (Falco rufigularis) \\
Laurel (Cordia alliodora) & Chacha (Ortalis vetula) \\
Rosul (Dalbergia sp.) & Lechuza (Otus guatemalae) \\
& Rey zope (Sarcoramphus papa) \\
& Garza tigre (Trigrisoma mexicanus) \\
\hline
\end{tabular}

Las formas más habituales de explotación de los recursos naturales son la captura y venta de especies animales, que sirven de mascotas o para fines médicos, de adorno, o por el valor de su carne, tanto para consumo propio como para su venta. También es habitual la tala de árboles que se destinan a abastecer a las grandes empresas madereras del área y también se suelen utilizar para la construcción de las casas lujosas que existen en el entorno.

Paralelamente a esta explotación de los recursos naturales, se intensificó la agricultura para cubrir, no sólo sus propias necesidades, sino también producir excedentes que se destinan a la venta en los mercados de Livingston, la localidad más próxima. El agro-sistema itinerante basado, principalmente, en el cultivo de maíz y frijol, se practica en un medio ecológicamente frágil donde, a la baja fertilidad de los suelos por lixiviación de los nutrientes hay que unir la topografía accidentada, lo que genera graves problemas de erosión. La intensificación de la agricultura ha consistido, por una parte, en el uso de abonos químicos y herbicidas $y$, por otra, en la reducción del tiempo de barbecho. 
El abono químico más frecuente es el Triple Quince (NPK). Las aplicaciones de este fertilizante sobre el suelo están generando graves problemas de regeneración de la vegetación autóctona en los períodos de barbecho, debido a que provoca en el suelo importantes desequilibrios en la disponibilidad de nutrientes para las plantas. Entre los herbicidas más usados se encuentra el Gramoxone, que está especialmente indicado para el control de las malas hierbas del maíz, café, cacao y caña de azúcar, entre otros cultivos. Estos productos son altamente contaminantes. Al mismo tiempo, estos compuestos se utilizan de manera incorrecta, debido a que sus usuarios, o bien son analfabetos o bien no entienden las instrucciones del etiquetado, por lo que las concentraciones de producto que se utilizan suelen ser más elevadas que las recomendadas. Entre los efectos más importantes, cabe destacar que destruyen a los microorganismos del suelo, con lo que dificultan aún más la regeneración de la vegetación natural. También afecta a los insectos que antes se alimentaban de las malas hierbas, ya que ahora los herbicidas destruyen su alimentación, por lo que éstos atacan a las plantas de maíz. En la actualidad hay graves problemas de este tipo en Guatemala con un insecto conocido como Gallina Ciega.

Con respecto al periodo de barbecho, hay que indicar que éste se ha reducido considerablemente, ya que se ha pasado de 6 u 8 años de media, a aproximadamente 4 ó 5 años, llegando en algunos casos hasta los 3 años. La causa es un proceso complejo que se originó con el aumento de la población y, por lo tanto, con la necesidad de más espacio para cultivar, lo que ha chocado frontalmente con la falta de nuevas tierras, debido a la privatización que han sufrido muchas áreas de la selva y al hecho de estar incluidas muchas aldeas en espacios naturales protegidos, donde está prohibida la roturación de nuevas tierras. Todo esto ha provocado la reducción del período de descanso de las tierras de cultivo como única posibilidad de alimentar a esta población creciente.

Este fenómeno de aumento de la población que está teniendo lugar actualmente en las áreas rurales de Guatemala, responde a un hecho generalizado que ya se ha constatado en otras áreas del planeta que tienen como denominador común, el hecho de estar circunscritas al ámbito agrícola itinerante. Por ejemplo, en Nagaland, al NE de La India (MOPT, 1989), se constató hace ya más de una década el mismo proceso de aumento de la población, lo que ha traído las mismas consecuencias: ruptura del ciclo agrícola tradicional y la consiguiente degradación del medio al producirse sobre un ecosistema frágil.

\subsection{La planificación del proyecto de ecoturismo}

El proyecto de ecoturismo que está desarrollando la Asociación Ak' Tenamit en la aldea Plan Grande Quehueche se estructura en dos grandes fases. En la primera, el objetivo es aplicar un modelo de proyecto ecoturístico sobre la citada aldea que se tomaría como área piloto de experimentación. Los resultados econó- 
micos y ambientales obtenidos servirán para evaluar el éxito del proyecto y la posibilidad de trasladar el modelo a otras aldeas del área, proceso que se corresponde con la segunda fase del proyecto.

El proyecto se encuentra actualmente en la primera fase de su desarrollo. La metodología de trabajo y los resultados obtenidos son los aspectos que se van a analizar seguidamente.

Para la planificación del proyecto se partió, primeramente, de la experiencia de los diversos proyectos comunitarios de ecoturismo que existían en la región. Ninguno de ellos podía considerarse especialmente exitoso, a pesar de los múltiples atractivos de la zona. Los problemas que se detectaron fueron diversos, entre los más importantes destacan que la capacitación del personal y las infraestructuras eran pésimas, los verdaderos beneficiarios de los proyectos no eran las comunidades, la publicidad y el marketing o eran deficientes o no existían y, por último, en unos casos los proyectos simplemente estaban mal ubicados y, en otros, las supuestas tradiciones que mostraban eran falsas, por lo que se estaba engañando al turista.

A continuación, se realizó el proceso de selección de la aldea piloto donde se iba a desarrollar el proyecto ecoturístico. Se partió de 26 aldeas, repartidas por 4 Espacios Naturales Protegidos del Departamento de Izabal: Parque Nacional Río Dulce, Biotopo Chocón-Machacas, Área de Protección Especial Río Sarstún y la Reserva de Manantiales Cerro San Gil (Figura 1). El objetivo de este estudio inicial fue seleccionar la aldea que reuniese las condiciones más idóneas para la aplicación del modelo de proyecto ecoturístico. Después de un largo proceso que duró aproximadamente un año y medio, concretamente entre septiembre de 1999 y marzo de 2001, se seleccionó a la aldea maya Plan Grande Quehueche, enclavada en el Área de Protección Especial Río Sarstún, para desarrollar el proyecto. Los motivos fueron varios. Comparativamente con el resto de las aldeas, Plan Grande Quehueche está mejor organizada, conserva mejor sus tradiciones mayas y no existen conflictos graves dentro de la misma, como la lucha por la tierra. Al mismo, tiempo la aldea está enclavada en la misma selva, lo que es un requisito indispensable por su mayor atractivo turístico y, además, está relativamente bien comunicada porque se puede llegar caminando, lo que abarata los costes de transporte al no tener que utilizar lanchas a través de los numerosos ríos del área para llegar hasta ella. La aldea cuenta además, con un bosque de gran extensión y bien conservado, a pesar de que aquí se encontraba el mayor número de furtivos de la zona, por lo que era la aldea que infringía un mayor impacto sobre la fauna en peligro de extinción del área. Por lo tanto, era una de las aldeas donde más urgía la diversificación económica para dar nuevas alternativas de ingresos y reducir la presión sobre el medio.

La aldea pertenece al municipio de Livingston (Departamento de Izabal, Guatemala). Desde el punto de vista fisiográfico, la aldea se encuentra en la cuenca del Río Quehueche, cuyas aguas desembocan en el Mar Caribe, concretamente en la 
Bahía de Amatique (Figura 1). El relieve de la aldea es alomado y fuertemente compartimentado, con pendientes que varían del 15 al $40 \%$ y unas laderas generalmente cortas, entre 300 y 500 metros de longitud. El sustrato litológico está compuesto por aluviones cuaternarios no consolidados de arenas, limos y gravas de cuarcitas y calizas, entre los que se intercalan lentes de arcillas y material orgánico de origen deltaico. Estos sedimentos descansan sobre un sustrato calcáreo con intercalaciones de lutitas. El clima de la zona es tropical húmedo con unas precipitaciones medias en torno a los $2.000 \mathrm{~mm}$ anuales y una temperatura media de $27^{\circ} \mathrm{C}$, con un máximo de $32{ }^{\circ} \mathrm{C}$ y un mínimo de $22^{\circ} \mathrm{C}$. El bosque tropical húmedo es la formación vegetal del área en la que se encuentra la aldea. Los suelos son de escasa fertilidad, debido a que las condiciones bioclimáticas del área favorecen la descomposición de la materia orgánica, lo que unido a las elevadas precipitaciones provocan serios problemas de lixiviación de nutrientes en el suelo. A este problema, hay que unir la elevada pendiente del entorno de la aldea, así como la deforestación a causa del cultivo de tierras. Esta situación crea las condiciones necesarias para que exista una elevada susceptibilidad a la erosión, siendo frecuentes la formación de surcos y cárcavas después de los períodos de lluvias.

El proyecto ecoturístico sólo suscitó el interés de 19 de las 60 familias que constituyen la aldea. Durante los meses restantes de 2001 se prosiguió con la planificación del proyecto dentro de la aldea. Se comenzó formando a las 19 familias que integraban el proyecto en temas muy variados como: educación ambiental, servicio al cliente, higiene y elaboración de alimentos, contabilidad, servicio de guía y gestión de proyectos. Paralelamente, se empezó a construir toda la infraestructura necesaria para la atención óptima del turista, lo que incluía una vivienda de cuatro habitaciones dobles, conservando en todo lo posible la arquitectura popular, dos duchas y un aseo tipo letrina abonera seca, que será completado próximamente con otro con agua y fosas sépticas.

Cater (1993) indica que el ecoturismo puede incluir aspectos de insostenibilidad, si no se realiza una adecuada planificación y manejo de los mismos proyectos, lo que incluye la implementación de medidas dirigidas a la prevención de impactos. Con este objetivo, en la aldea se llevaron a cabo diversas medidas de prevención para minimizar los posibles impactos tanto ambientales como culturales. Teniendo en cuenta que la aldea presenta una población compuesta por 60 familias, se estimó que sería conveniente limitar las visitas turísticas, para lo cual se estableció una carga de visitantes de un máximo de 8 diarios y no más de 30 al mes, lo que eleva la carga máxima anual a un total de 360 visitas. Al mismo tiempo, se decidió hacer el proyecto más exclusivo y dirigido a un turista más selecto. Para ello, se proporcionaron mejores servicios e infraestructuras que en otros proyectos similares del entorno y se aumentó el precio del paquete turístico.

Los precios por persona y día son de aproximadamente 29 euros al cambio, incluyendo el alojamiento, las comidas y un paquete de actividades, entre las que destacan el senderismo por la selva, visita de cuevas, lagunas y cataratas, identi- 
ficación de plantas medicinales de la cultura maya, manifestaciones de música tradicional, cursos de artesanía y ceremonias mayas. Este precio puede parecer bajo pero es de los más elevados del país, aunque comparado con otros proyectos similares, a nivel internacional, resulta un precio muy competitivo.

Preocupados también por el posible impacto cultural que los flujos de turismo pudieran tener sobre los jóvenes de la aldea, éstos se beneficiaron de becas de estudio en el Instituto de Enseñanza Media de Ak' Tenamit, donde la recuperación y revalorización de la cultura maya son los ejes principales de la educación. Además se está trabajando también con los adultos en estos aspectos. Para afianzar sus tradiciones y creencias, la aldea cuenta con 2 sacerdotes mayas que han sido apoyados por la Asociación para participar en eventos y cursos relacionados con su cultura. Al mismo tiempo, se organizan en la aldea danzas y ceremonias mayas continuamente, sin la presencia de turistas, para que sus valores no se pierdan.

\subsection{Los resultados económicos del proyecto}

El proyecto de ecoturismo en la aldea Plan Grande Quehueche abrió sus puertas a los visitantes en enero de 2002. Los resultados económicos de los 2 primeros años son más que aceptables y las previsiones para el año 2004 son muy halagüeñas (cuadro 2).

Durante el año 2002 se recibieron un total de 75 visitantes. Los ingresos ascendieron a 1.910 euros netos que, una vez descontados los gastos, representó unos ingresos por familia de 101 euros. Los resultados pueden parecer exígüos por si mismos, pero no lo son si se tiene en cuenta que la renta per cápita anual por familia es de aproximadamente 156 euros. Por lo tanto, los ingresos por turismo representaron el $64,7 \%$ de la renta familiar. Estos datos se pueden considerar muy salisfactorios, teniendo en cuenta que son los resultados del primer año de proyecto y además, no se contaba con los operadores de turismo que actualmente tienen entre sus ofertas esta actividad.

Durante el año 2003 los resultados mejoraron sustancialmente y sin necesidad de subir los precios. El número de visitantes fue de 122, lo que generó unos ingresos que ascendieron a 2.720 euros netos. Por lo tanto, a cada familia le correspondieron 143 euros, lo que significa el $91,6 \%$ de la renta familiar, teniendo 156 euros como base 100 .

Para el año 2004 los resultados se prevén todavía más prometedores. Las expectativas que se esperan, en base a las reservas ya realizadas por diferentes particulares y agencias de viajes, son un incremento en el número de visitantes de alrededor del $52 \%$, lo que supone un número de visitas cercano a las 200 . Los ingresos netos ascenderían a unos 4130 euros aproximadamente, correspondiéndole a cada familia unos 217 euros, lo que representa el $139,1 \%$ de la renta familiar. 
Cuadro 2. Resultados económicos del proyecto de ecoturismo

\begin{tabular}{c|c|c|c|c}
\hline Año & $\begin{array}{c}\text { Nümero } \\
\text { de visitas }\end{array}$ & $\begin{array}{c}\text { ingresos netos } \\
\text { (euros) }\end{array}$ & $\begin{array}{c}\text { Ingresos/familia } \\
\text { (euros) }\end{array}$ & $\begin{array}{c}\text { Ingresos/renta } \\
\text { familiar (\%) }\end{array}$ \\
\hline 2002 & 75 & 1.910 & 101 & 64,7 \\
\hline 2003 & 122 & 2.720 & 143 & 91,6 \\
\hline Previsión para 2004 & 200 & 4.130 & 217 & 139,1 \\
\hline
\end{tabular}

FUENTE: Estadísticas Asociación Ak' Tenamit.

Teniendo en cuenta estos resultados, la actividad turística que se desarrolla en la aldea es muy rentable, pero no sólo por los ingresos que se han obtenido, sino también por la productividad del trabajo invertido. Las tareas que desempeñan las familias de la aldea, en relación al turismo, están divididas por funciones y cada una de ellas es llevada a cabo por familias diferentes. De manera que, con una visita, una familia se encarga de la comida, otra del servicio de guía en la naturaleza, otra de las actividades culturales y otra de la limpieza y mantenimiento de la vivienda. Este reparto de las tareas, teniendo en cuenta que el número de visitantes es aún bajo y son 19 las familias que están implicadas, provoca que la mayoría de estas familias no trabajen más de 12 días al año en total, considerando una jornada diaria de trabajo de 8 horas. Comparativamente con el cultivo del maíz, estas mismas personas trabajan una media de 237 días y obtienen 156 euros, por lo que con esta nueva actividad ya están consiguiendo un $91,6 \%$ de las ganancias que la agricultura les proporciona, trabajando sólo un $5,1 \%$ del tiempo.

Por otra parte, el número máximo de visitantes permitido al año es de 360 , por lo que todavía pueden incrementar sus ingresos en un $66 \%$ más, aunque también su dedicación que se multiplicaría por 3 , pero aún así tan sólo ascendería a unos 36 días al año.

Cada año se van haciendo contactos con nuevos operadores de turismo nacionales e internacionales interesados en este tipo de oferta y, además, se van afianzando las relaciones con las empresas que ya ofrecen este servicio turistico. La Asociación cuenta con 2 portales en internet con el que se puede acceder a los programas de ecoturismo, www.aktenamit.org y www.projectguatemala.org/aktenamit. Pese a todo, son pocos los visitantes que acceden a este servicio a través de este sistema, siendo el turismo organizado en circuitos a partir de operadores turísticos el que accede a la aldea, en contra de la norma general de los proyectos de este tipo.

El perfil medio del visitante que viene a disfrutar del ecoturismo en la aldea es de origen europeo, principalmente españoles y franceses, y después le siguen los estadounidenses. La mayor parte son de clase media, suelen hablar español, su edad está comprendida entre los 30 y 45 años y suelen quedarse una sola noche, aunque el porcentaje que permanece dos noches va en aumento. Suelen ser per- 
sonas muy interesadas por la naturaleza, pero aún más por las culturas ancestrales. Normalmente el nivel educativo de estos visitantes es alto, generalmente universitario.

\section{CONCLUSIONES}

Los resultados económicos del proyecto, aunque son bajos en términos absolutos, se pueden valorar como muy exitosos si son matizados por las circunstancias socioeconómicas y medioambientales de la comunidad. Los ingresos del ecoturismo han tenido una repercusión importante en el nivel de vida de las familias integrantes del proyecto que, aunque no han salido todavía del umbral de pobreza, sus necesidades no cubiertas han disminuido. Estos ingresos, junto con el de otros proyectos productivos, han conseguido que la extensión de los maizales de estas familias se haya reducido en dos años entre un 20 y un $25 \%$, lo que supone 16 hectáreas de bosque húmedo tropical salvado cada año. No ha sido necesario hablar con las familias sobre la reducción de estas extensiones, ellas por iniciativa propia se han dado cuenta de la importancia de disminuir los maizales para conservar los recursos vegetales. De la misma manera, la aldea ha pasado de ser la principal cazadora de jaguar en el área, a estar orgullosa de haber cesado esta actividad, aprovechando ahora a esta especie animal por su atractivo turístico.

Las claves del éxito del proyecto son varias. Por una parte, hay que destacar el hecho de no haber creado un sentimiento de paternalismo en la aldea. Aunque las infraestructuras y parte de las capacitaciones de los integrantes del proyecto tienen su origen en el Proyecto RECOSMO, cuyos fondos provienen de donaciones destinadas a la cooperación por parte de Holanda y el Fondo Mundial para el Medio Ambiente (GEF), las familias implicadas tuvieron que aportar su mano de obra: transporte de todos los materiales de construcción a través de 8 kilómetros de selvá, construcción de toda la infraestructura, e incluso tuvieron que proporcionar su mano de obra como apoyo a otras obras sociales que Ak' Tenamit tiene en el área. De esta manera se evitó que la donación se considerara un regalo, ya que estas personas a partir de su trabajo sienten que toda la infraestructura y el proyecto les pertenece, por lo que sienten la necesidad de cuidarlo y explotarlo al máximo.

Por otra parte, el proyecto de ecoturismo aporta como novedad que la comunidad, después de sólo 2 años de trabajo, ejerce sobre el mismo un elevado control, sin apenas dependencia de Ak' Tenamit, salvo en el proceso de captación y reserva de los turistas. Este hecho asegura la continuidad en el tiempo del proyecto, al no ser necesario un apoyo externo indefinido. Este factor ha sido una de las causas del fracaso de muchos proyectos en toda Latinoamérica, en los que al cesar el apoyo externo, las comunidades no han sabido cómo continuarlos.

Este proyecto de ecoturismo ha recibido un importante respaldo a través del reconocimiento, tanto nacional como internacional, de los logros alcanzados. La Red de Turismo Sostenible de Guatemala declaró a Plan Grande Quehueche, durante el 
II Congreso de Turismo Sostenible de Guatemala, como el mejor proyecto de turismo sostenible del país. Este premio fue otorgado en octubre de 2002, sólo 10 meses después de que el proyecto abriera sus puertas. Asimismo, durante el año 2003 recibió diferentes menciones. La Organización Internacional del Trabajo (OIT) destacó el proyecto como uno de los tres mejores proyectos de turismo comunitario de Guatemala, y publicó un estudio de caso sobre la experiencia. La Organización Mundial de Turismo (OMT), en su publicación mundial sobre Mejores Prácticas, destacó el proyecto como el único ejemplo de Guatemala y uno de los tres ejemplos en toda Centroamérica en el que se estaban llevando a cabo prácticas adecuadas de ecoturismo (OMT, 2003). Por último, la Unión Mundial para la Naturaleza (UICN) declaró el proyecto en el 2003 como uno de los tres mejores a nivel mundial, por sus beneficios para el medio ambiente y por el modo en que las comunidades han sido involucradas en su gestión (Calvo, 2003), grabando un vídeo que fue mostrado durante el Congreso Mundial de Áreas Protegidas de Sudáfrica de ese año.

El proyecto ecoturístico de Plan Grande Quehueche es, por lo tanto, un ejemplo de proyecto productivo sostenible desde el punto de vista económico y medioambiental. Es además un proyecto con grandes capacidades de adaptación a otros espacios de América Latina, siempre y cuando se tengan en cuenta la cultura, el medio ambiente y la situación coyuntural de cada área, porque no se debe olvidar que ninguna experiencia de este tipo se puede aplicar a otros espacios, siguiendo un modelo rígido.

\section{BIBLIOGRAFIA}

Bustos, P. (2003): Jardín Refugio del Colibri en urbanización La Armenia. Universidad de Especialidades Turísticas de Quito (Ecuador). Tesis de Licenciatura.

BuRgER, J. (2000): «Landscapes, tourism and conservation». The Science of the Total Environment, 249 , 39-49.

CALVO, M. (2003): We all have the right to work. Unión Mundial para la Naturaleza (UICN).

CATER, E. (1993): «Ecotourism in the Third World: problems for sustainable tourism development". Tourism Management, 14(2), 85-90.

Farrell, B. H. and RunYan, D. (1991): «Ecology and tourism». Annales of Tourism Research, 18(1), 2640.

FOUCAT, V.S.A. (2002): "Community-based ecotourism management moving towards sustainability in Ventanilla, Oaxaca, Mexico». Ocean Coastal Management, 45, 511-529.

GREIF, A. (1993): "Contract enforceability and economic institutions in early trade". American Economic Review, 83: 525-549.

GreIF, A. (1994): «Cultural beliefs and the organization of society: a historical and theorical reflection on collectivist and individualist societies". Journal of Political Economy, 102: 912-950.

Grenier, D., KaAe, B. C., Miller, M. L. and MOBley, R. W. (1993): “Ecotourism, landscape architecture and urban planning». Landscape and Urban Planning, 25 (1-2), 1-16.

HONEY, M. (1999): Ecotourism and Sustainable Development: Who owns paradise?. Island Press. Washington, $405 \mathrm{pp}$.

JACOME, F. y ACOSTA, J. (2003): Centro de Interpretación Cascada Chorro Blanco en comunidad Las Juntas. Universidad de Especialidades Turisticas de Quito (Ecuador). Tesis de Licenciatura.

LINDBERG, K. and ENRIQUEZ KEITH, J. (1996): "Ecotourism questioned. Case studies from Belize». Annals of Tourism Research, 23 (3), 543-562.

LUCK, M. and KIRSTAGES, T. (eds) (2002): Global ecotourism policies and case studies perspectives and constraints. Channel View Publications. Clevedon, $216 \mathrm{pp}$. 
Masberg, B. A. and Morales, N. (1999): "A case analysis of strategies in ecotourism development". Aquatic Ecosystem Health and Management, 2, 289-300.

MASON, P. (2003): Tourism impacts, planning and management. Butterworth-Heinemann. Amsterdam, 195 $\mathrm{pp}$.

MOPT (1989): El hombre pertenece a la Tierra. Comité Español MaB. UNESCO.

Morera BeIta, C. (2002): Ecoturismo, desarrollo local y conservación: el caso de la Peninsula de Osa, Costa Rica. Universidad de Barcelona. Tesis Doctoral.

NeLlo ANDREU, M.G. (2003): Ecoturismo, consenación de la naturaleza y desarrollo local. El caso de México, América Central y las Grandes Antillas. Universitat Rovira i Virgili. Tesis Doctoral.

Newsome, D., Moore, S. and Dowling, R. (2002): Natural Area Tourism: Ecology, Impacts and Management. Channel View Publications. Clevedon.

NovelLI, M. (2004): Niche Tourism. Butterworth-Heinemann, 288 pp.

OMT (2003): Desarrollo Sostenible del Ecoturismo: una Compilación de Buenas Prácticas. Organización Mundial de Turismo (OMT).

Orams, M. B. (1995): "Towards a more desirable form of ecotourism". Tourism Management, 16(1), 3-8.

Ross, S. and WALL, G. (1999): «Ecotourism: towards congruence between theory and practice». Tourism Management, 20, 123-132.

SCHEYVENS, R. (1999): “Ecotourism and the empowerment of local communities". Tourism Management, 20, 245-249.

Solano PaCheco, L. (2003): Experiencia Ambiental de Costa Rica en el Desarrollo y Posicionamiento del Ecoturismo. PNUD. (www.undp.org/surf-panama).

SteAM, C. (2001): El Papel del Desarrollo Local en el Manejo de un Área Protegida: un Caso de Estudio Comparativo de Ecoturismo en Costa Rica. Cornell University (USA). (www.eco-index.org).

WALLACE, G.N. and PIERCE, S. M. (1996): “An evaluation of ecotourism in Amazonas, Brasil». Annals of Tourism Research, 23(4), 843-873.

WEABER, D.B. (1988): Ecotourism in the Less Developed World. CAB International. U.K., 258 pp.

WEARING, S. and NEIL, J. (1999): Ecotourism: impacts, potentials and possibilities. Butterworth-Heinemann. Oxford, $163 \mathrm{pp}$. 